\title{
Chemical evolution models for the dwarf spheroidal galaxies Leo 1 and Leo 2
}

\author{
G. A. Lanfranchi ${ }^{1}$ and F. Matteucci ${ }^{2,3}$ \\ 1 Núcleo de Astrofísica Teórica, Universidade Cruzeiro do Sul, R. Galvão Bueno 868, Liberdade, 01506-000, São Paulo, SP, Brazil \\ e-mail: gustavo.lanfranchi@cruzeirodosul.edu.br \\ 2 Dipartimento di Astronomia-Universitá di Trieste, via G. B. Tiepolo 11, 34131 Trieste, Italy \\ 3 INAF Osservatorio Astronomico di Trieste, via G.B. Tiepolo 11, 34131, Italy
}

Received 31 July 2009 / Accepted 17 December 2009

\section{ABSTRACT}

\begin{abstract}
Aims. We investigate the chemical evolutionary history of the dwarf spheroidal galaxies Leo 1 and Leo 2 by means of predictions from a detailed chemical evolution model compared to observations. The model adopts up to date nucleosynthesis and takes into account the role played by supernovae of different types (Ia, II), allowing us to follow in detail the evolution of several chemical elements (H, $\mathrm{D}, \mathrm{He}, \mathrm{C}, \mathrm{N}, \mathrm{O}, \mathrm{Mg}, \mathrm{Si}, \mathrm{S}, \mathrm{Ca}, \mathrm{Fe}, \mathrm{Ba}$, and $\mathrm{Eu})$.

Methods. Each galaxy model is specified by the prescriptions of the star formation rate and by the galactic wind efficiency chosen to reproduce the main features of these galaxies, in particular the stellar metallicity distributions and several abundance ratios. These parameters are constrained by the star formation histories of the galaxies as inferred by the observed color-magnitude diagrams, indicating extended star formation episodes occurring at early epochs, but also with hints of intermediate stellar populations.

Results. The main observed features of the galaxies Leo 1 and Leo 2 can be very well explained by chemical evolution models according to the following scenarios: the star formation occurred in two long episodes at 14 Gyr and 9 Gyr ago that lasted 5 and $7 \mathrm{Gyr}$, respectively, with a low efficiency $\left(v=0.6 \mathrm{Gyr}^{-1}\right)$ in Leo 1 , whereas the star formation history in Leo 2 is characterized by one episode at $14 \mathrm{Gyr}$ ago that lasted $7 \mathrm{Gyr}$, also with a low efficiency $\left(v=0.3 \mathrm{Gyr}^{-1}\right)$. In both galaxies an intense wind (nine and eight times the star formation rate $-w_{i}=9$ and 8 in Leo 1 and Leo 2, respectively) takes place which defines the pattern of the abundance ratios and the shape of the stellar metallicity distribution at intermediate to high metallicities.

Conclusions. The observational constraints can only be reproduced with the assumption of gas removal by galactic winds.
\end{abstract}

Key words. Local Group - galaxies: evolution - galaxies: dwarf - galaxies: abundances

\section{Introduction}

Local dwarf spheroidal (dSph) galaxies have been the subject of a series of studies both from the observational and theoretical point of view in the past few years (Shetrone et al. 2003; Tolstoy et al. 2003; Venn et al. 2004; Lanfranchi \& Matteucci 2003, 2004; Bonifacio et al. 2004; Monaco et al. 2005; Geisler et al. 2005; Fenner et al. 2006; Helmi et al. 2006; Marcolini et al. 2006; Koch et al. 2006; Sbordone et al. 2007; Shetrone et al. 2009; Revaz et al. 2009). From all these works emerged a scenario in which these galaxies are characterized by low metallicities, a sharp decrease of $[\alpha / \mathrm{Fe}]$ ratios at high metallicities, and by a stellar metallicity distribution (SMD) with a peak at low $[\mathrm{Fe} / \mathrm{H}]$, a low number of metal-poor stars, and a sharp decline at the high-metallicity tail. Besides that, the results from colormagnitude diagrams (CMD) indicate that these galaxies exhibit complex star formation histories, that differ from one system to another. Almost all of them show signs of an ancient population of stars, but several are also marked by intermediate populations and even by very recent star formation (Hernandez et al. 2000; Bellazzini et al. 2002; Carrera et al. 2002; Dolphin et al. 2005, and references therein). Carina for example probably suffered four episodes of star formation, two of them at early epochs (between 14 and 10 Gyr ago) and two more recently (Rizzi et al. 2003). Another striking feature of the dSphs is that they are almost totally depleted of neutral gas in their central regions.
What is the link between all these characteristics? How can all of them be joined in a consistent scenario for the formation and evolution of all the dSph galaxies? There is a general consensus that the removal of the gas content of the galaxy is the main factor in modeling the observed metallicity patterns and SMDs, and driving the evolution of the dSph galaxies. The mechanism responsible for the gas loss is, however, still a matter of debate. Is it an internal mechanism (such as galactic winds) or external (ram pressure, tidal stripping)? Several works have favored either one or the other suggestion (van den Bergh 1994; Burkert \& Ruiz-Lapuente 1997; Ferrara \& Tolstoy 2000; Fragile et al. 2003; Robertson et al. 2005). One way to approach this question is to search for isolated $\mathrm{dSph}$ galaxies and to investigate their evolution. If isolated $\mathrm{dSph}$ galaxies exhibit chemical properties similar to those near large galaxies, the argument that the gas loss is caused by an internal mechanism like galactic winds, would be strengthened (see also Shetrone et al. 2009). One should keep in mind though, that even the more distant $\mathrm{dSph}$ galaxies are not free of any interaction with the Galaxy in the past. Tidal interactions of the Milky Way with other galaxies (Mayer et al. 2001; Kroupa et al. 2005; Metz et al. 2009) and resonant stripping have been suggested as possible scenarios for the formation of local dSph galaxies. In this case, even galaxies with large galactocentric distances could have had their evolution affected by external factors which would have removed a large fraction of their gas content. 
The dSph galaxies Leo 1 and Leo 2 are excellent objects to perform the analysis of isolated systems. They are among the most distant dSph satellites in the Milky Way system $(\sim 270 \mathrm{Kpc}$ and $204 \mathrm{Kpc}$, respectively) and probably free from any presentday dynamical influence from the Milky Way. To understand the processes which played a major role on the evolution of these two galaxies would provide very important clues in the study of dSph galaxies as a whole. Similar to other nearby dSph galaxies, they are characterized by low metallicities and exhibit the same $\alpha$-element deficiency at high metallicities. Besides that, the metallicity peak of their main stellar populations in the SMDs is located at low $[\mathrm{Fe} / \mathrm{H}](\sim-1.4$ dex and -1.6 dex in Leo 1 and Leo 2, respectively) followed by a steep decline at the high metallicity tail (Bosler et al. 2007; Koch et al. 2007a,b; Gullieuszik et al. 2009). Color-magnitude studies indicate that they both formed stars in the early epochs of their evolution, but in different fractions. Leo 2 formed the majority of its stars 7-14 Gyr ago, whereas the major episode of star formation in Leo 1 occurred between 1-7 Gyr ago with a minor fraction of stars being formed before $\sim 10 \mathrm{Gyr}$ ago.

In this work we adopt detailed chemical evolution models with the aim to provide a consistent scenario for the evolution of these two galaxies. This scenario should allow us to model and reproduce the above observational features. We follow the same approach as in our previous papers regarding other local dSph galaxies (Lanfranchi \& Matteucci 2003, 2004; and Lanfranchi et al. 2006a, 2008). The model for each galaxy is determined mainly by the star formation rate (SFR) and galactic wind efficiency prescriptions, chosen to reproduce the main observational constraints. In our model, when the thermal energy of the interstellar medium (ISM) is equal or higher than the binding energy of the galaxy, a galactic wind occurs, thus removing a considerable fraction (depending on the wind efficiency) of the gas content of the system. This wind is also responsible for a considerable decrease in the star formation (SF) of the galaxy in the first Gyr of its evolution. In Lanfranchi \& Matteucci (2003, 2004 - LM03, LM04) we adopted high values for the wind efficiency (from 5 to 10 times the SFR) to reproduce the abundance ratios and the observed present day gas fraction of each galaxy, given that our models do not assume any external removal of gas. Later works with the same models (with no modifications in the main parameters) allowed us also to reproduce the metallicity distribution of the same dSph galaxies (Carina, Sagittarius, Draco, and Ursa Minor - Lanfranchi et al. 2006b; Lanfranchi \& Matteucci 2007 - LM07) and the abundance ratios of neutron capture elements (Lanfranchi et al. 2006a - LMC06a). The galaxies so far analyzed are close to our Galaxy and consequently subject to tidal and dynamical effects. Leo 1 and Leo 2, on the other hand, are at a large galactocentric distance, therefore any later removal of gas could be caused by an internal mechanism like galactic winds.

Different approaches were adopted in the study of these galaxies leading to scenarios with similarities but also discrepancies compared to LM03 and LM04 (see Lanfranchi et al. 2007, for more details). In particular, Carigi et al. (2002), Ikuta \& Arimoto (2002) and Fenner et al. (2006) also adopted chemical evolution models in their analysis of local dSph galaxies. Neither one of them, however, compared their results with the stellar metallicity distributions observed, which LM consider one of the strongest constraint in the chemical evolution studies, specially when one is analyzing the effects of the galactic winds on the evolution of these galaxies. Besides that, Carigi et al. (2002) and Ikuta \& Arimoto (2002) compared their results to a much more limited sample of stars and did not consider the removal of metals by galactic winds. Fenner et al. (2006) on the other hand adopted a more detailed chemical evolution model compared to a more complete data sample. They argue that the evolution of several abundance ratios could be reproduced by models with moderate galactic winds (less efficient than LM04) which are not able to remove the remaining gas content of the galaxy. They conclude then that an external mechanism should be acting togheter with the winds. They did not compare their results to the stellar metallicities distribuitions of these galaxies however.

More recently, Revaz et al. (2009) followed a completely different approach. By means of hydrodynamical Nbody/Tree-SPH simluations they studied the evolution of isolated dSph galaxies. The initial total mass is the main driver of the system in their simulation, contrary to LM03 and LM04 in which the SF and wind efficiencies play the major role. Since the final gas mass predicted in their simulations is much higher than the values inferred by observations, they claim the necessity of invoking external processes to remove the gas content that remains at the end of the SF. They also compared their predictions to the $[\mathrm{Mg} / \mathrm{Fe}]$ ratio and the stellar metallicity distribution observed in a few local dSph galaxies. Their models, however, are not capable of reproducing the lowest values of $[\mathrm{Mg} / \mathrm{Fe}]$ as precisely as the higher ones. In fact, the majority of stars show an almost linear trend of $[\mathrm{Mg} / \mathrm{Fe}]$ as a function of $[\mathrm{Fe} / \mathrm{H}]$ (the green to blue areas in their Fig. 12). Besides that, their SMD tend to have a peak at metallicities higher than the ones observed even with the shift of a few tenths of dex. As they have not tried to fit any galaxy in particular (contrary to LM04 goal), these discrepancies are not taken into account. In the LM models, the lowest values observed of $[\alpha / \mathrm{Fe}]$ are explained by the effects of intense winds on the star formation rate. A more direct comparison between the LM scenarios with theirs is difficult because the main parameters in each simulation are quite different. LM04 adopt for instance the star formation histories (SFH) inferred from color-magnitude diagrams for each galaxy as an input of the models, whereas in Revaz et al. (2009) the SFH is a consequence of the initial parameters of the simulation. The parameters representing the efficiency of the SF are also different, which prevents a more complete comparison.

The paper is organized as follows: in Sect. 2 we present the observational data concerning the Leo 1 and Leo 2 dSph galaxies, in Sect. 3 we describe the adopted chemical evolution models and theoretical prescriptions, in Sect. 4 we describe the results of our models, and finally in Sect. 5 we discuss the results and draw some conclusions. We use the solar abundances measured by Grevesse \& Sauval (1998) when the chemical abundances are normalized to the solar values $([\mathrm{X} / \mathrm{H}]=\log (\mathrm{X} / \mathrm{H})-$ $\left.\log (\mathrm{X} / \mathrm{H})_{\odot}\right)$.

\section{Data sample}

The observed data collected in this work and used as main constraints for the comparison with the model predictions are based on particular abundance ratios and stellar metallicity distributions. Abundance ratios are powerful tools in the study of the chemical evolution of galaxies because they depend mainly on the nucleosynthesis prescriptions, stellar lifetimes and adopted initial mass function (IMF), and not on the other model parameters. The stellar metallicity distributions, on the other hand, are representative of the chemical enrichment of the galaxy and provide information about the history of the chemical evolution and how it proceeded (LM04). Hence, these two observables together provide strong constraints on chemical evolution models 
and limit the range of acceptable values for several other model parameters.

We compared the predictions of the model with $[\alpha / \mathrm{Fe}],[\mathrm{r}$, $\mathrm{s} / \mathrm{Fe}]$, and the stellar metallicity distributions observed in both galaxies. For Leo 1 we used the abundance data from Shetrone et al. (2003) with the update from Venn et al. (2004). In this case the abundance ratios include $[\alpha / \mathrm{Fe}],[\mathrm{Ba} / \mathrm{Fe}]$, and $[\mathrm{Eu} / \mathrm{Fe}]$, but only for two stars. Even though the sample is limited, it is enough to complement the comparison with the metallicity distribution. Many more stars were observed in Leo 2 (almost 30 ), but only $[\mathrm{Mg} / \mathrm{Fe}]$ and $[\mathrm{Ca} / \mathrm{Fe}]$ were inferred (Shetrone et al. 2009). For this galaxy the analysis of the abundance ratios is also complemented by the metallicity distribution. The observed stellar metallicity distributions for both galaxies were taken from Koch et al. (2007a - Leo 1, 2007b - Leo 2), Bosler et al. (2007), and Gullieuszik et al. (2009). In the papers from Koch et al. (2007a,b) and Gullieuszik et al. (2009) the abundance of iron $([\mathrm{Fe} / \mathrm{H}])$ is inferred from the Ca triplet lines $(\mathrm{CaT})$. One should take care though when such a procedure is adopted in extragalactic stars. The calibration of the Ca II lines and the transformation into $\mathrm{Fe}$ abundance contains several uncertainties. For example, calcium and iron are formed in totally different nucleosynthesis processes and consequently do not trace each other directly. One should therefore take into account the variations of $[\mathrm{Ca} / \mathrm{Fe}]$ in the course of the evolution of the galaxy in the calibration. Normally this is done for Galactic globular cluster stars, which do not share the same star formation histories and abundance ratio patterns as the stars in $\mathrm{dSph}$ galaxies. In order to avoid the fundamental dependence on $[\mathrm{Ca} / \mathrm{Fe}]$ ratios built into $\mathrm{CaII}$ calibrations, Bosler et al. (2007) adopted also $[\mathrm{Ca} / \mathrm{H}]$ as a metallicity indicator. In this work both the metallicity distribution as a function of both $[\mathrm{Fe} / \mathrm{H}]$ and $[\mathrm{Ca} / \mathrm{H}]$ are compared to the predictions of the models.

\section{Models}

In order to study the chemical enrichment in Leo 1 and Leo 2 $\mathrm{dSph}$ galaxies, we adopted the same models as in previous works (LM04). These models adopt up-to-date nucleosynthesis yields for intermediate-mass stars (IMS) and supernovae (SNe) of both types (type Ia and type II) as well as the effects of SNe and stellar winds on the energetics of the interstellar medium. They are able to reproduce very well several observational constraints of six local dSph galaxies (in particular, Carina, Draco, Sagittarius, Sextan, Sculptor, and Ursa Minor), like [ $\alpha / \mathrm{Fe}]$, [s-r/Fe], the stellar metallicity distributions, and the present day gas mass and total mass. The scenario adopted for the formation and evolution of these galaxies considers long episodes of star formation with low rates and the occurrence of very intense galactic winds. As shown in LM03 and LMC06a, the low SFR is required to account for the low values of $[\alpha / \mathrm{Fe}]$ ratios, whereas the observed stellar metallicity distribution cannot be reproduced without invoking strong and efficient galactic winds (LM07).

The evolution of the abundances of several chemical elements $(\mathrm{H}, \mathrm{He}, \mathrm{C}, \mathrm{O}, \mathrm{Mg}, \mathrm{Si}, \mathrm{S}, \mathrm{Ca}, \mathrm{N}, \mathrm{Fe}, \mathrm{Ba}, \mathrm{La}, \mathrm{Eu}, \mathrm{Y}$, and others) can be followed in detail by the model, starting from the matter reprocessed by the stars and restored into the ISM by stellar winds and type II and Ia supernova explosions. The main characteristics of the models are: it is a one zone model with instantaneous and complete mixing of gas inside this zone; no instantaneous recycling approximation (i.e. the stellar lifetimes are taken into account) is adopted. The nucleosynthesis prescriptions are the same as in Lanfranchi, Matteucci and Cescutti (2008 - LMC08). In particular we adopted the yields of
Nomoto et al. (1997) for type Ia supernovae, Woosley \& Weaver (1995) (with the corrections suggested by François et al. 2004) for massive stars $\left(M>10 M_{\odot}\right)$, van den Hoek \& Groenewegen (1997) for intermediate mass stars (IMS), and the ones described in Cescutti et al. (2006, 2007) and Busso et al. (2001) for $s$ and $r$ process elements.

The type Ia SN progenitors are assumed to be white dwarfs in binary systems according to the formalism originally developed by Greggio \& Renzini (1983) and Matteucci \& Greggio (1986). The prescriptions for the SF (which follow a Schmidt law Schmidt 1963), initial mass function (IMF - Salpeter 1955), infall, and galactic winds are the same as in LM03 and LM04. The main parameters adopted for the model of each galaxy can be seen in Table 1, where $v$ is the star-formation efficiency, $w_{i}$ the wind efficiency, $n, t$, and $d$ are the number, time of occurrence, and duration of the SF episodes, respectively. Besides that also the predicted total luminous mass and the present day gas mass (Cols. 7 and 8, respectively) are shown.

In our scenario, the dSph galaxies form through a continuous infall of pristine gas until a luminous mass of $\sim 10^{8} M_{\odot}$ is accumulated. One crucial feature in the evolution of these galaxies is the occurrence of galactic winds, which develop when the thermal energy of the gas equals its binding energy (Matteucci \& Tornambé 1987). This quantity is strongly influenced by assumptions concerning the presence and distribution of dark matter (Matteucci 1992). A diffuse $\left(R_{\mathrm{e}} / R_{\mathrm{d}}=0.1\right.$, where $R_{\mathrm{e}}$ is the effective radius of the galaxy and $R_{\mathrm{d}}$ is the radius of the dark matter core $)$ but massive $\left(M_{\text {dark }} / M_{\text {Lum }}=10\right)$ dark halo has been assumed for each galaxy. The effects of changing the dark matter and its distribution in dwarf galaxies were explored in Bradamante et al. (1998). A larger dark matter halo and/or a more concentrated dark matter distribution will make the occurrence of the wind more difficult and therefore more metal rich stars will be predicted in the stellar metallicity distributions. Besides that, the sharp decrease observed in the abundance ratios would take place at higher metallicities than observed since this decrease is associated to the onset of the wind. With a larger/more concentrated dark matter halo the wind would take longer to develop, since more thermal energy would be required.

\subsection{Theoretical prescriptions}

The basic equation that describes the evolution in time of the fractional mass of the element $i$ in the gas within a galaxy, $G_{i}$, is the same as described in Tinsley (1980) and Matteucci (1996,b):

$\dot{G}_{i}=-\psi(t) X_{i}(t)+R_{i}(t)+\left(\dot{G}_{i}\right)_{\text {inf }}-\left(\dot{G}_{i}\right)_{\mathrm{out}}$,

where $G_{i}(t)=M_{\mathrm{g}}(t) X_{i}(t) / M_{\mathrm{tot}}$ is the gas mass in the form of an element $i$ normalized to a total fixed mass $M_{\text {tot }}$ and $G(t)=$ $M_{\mathrm{g}}(t) / M_{\text {tot }}$ is the total fractional mass of gas present in the galaxy at the time $t$. The quantity $X_{i}(t)=G_{i}(t) / G(t)$ represents the abundance by mass of an element $i$, with the summation over all elements in the gas mixture equal to unity. $\psi(t)$ is the fractional amount of gas turning into stars per unit time, namely the SFR. $R_{i}(t)$ represents the returned fraction of matter in the form of an element $i$ that the stars eject into the ISM through stellar winds and supernova explosions; this term contains all the prescriptions concerning the stellar yields and the supernova progenitor models. The infall of external gas and the galactic winds are accounted for by the two terms $\left(\dot{G}_{i}\right)_{\text {inf }}$ and $\left(\dot{G}_{i}\right)_{\text {out }}$, respectively. The prescription adopted for the star formation history is the main feature which characterizes the dSph galaxy models. 
Table 1. Models with galactic winds for the dSph galaxies Leo 1 and Leo 2.

\begin{tabular}{ccccccccc}
\hline \hline Galaxy & $v\left(\mathrm{Gyr}^{-1}\right)$ & $w_{i}$ & $n$ & $t(\mathrm{Gyr})$ & $d(\mathrm{Gyr})$ & $M_{\mathrm{tot}}\left(10^{7} M_{\mathrm{o}}\right)$ & $M_{\mathrm{gas}}\left(10^{4} M_{\mathrm{o}}\right)$ & $I M F$ \\
\hline Leo 1a & 0.15 & 6 & 2 & $0 / 5$ & $5 / 7$ & 2.15 & 1.85 & Salpeter \\
Leo 1b & 0.60 & 9 & 2 & $0 / 5$ & $5 / 7$ & 4.06 & 1.97 & Salpeter \\
Leo 1c & 1.70 & 10 & 2 & $0 / 5$ & $5 / 7$ & 8.33 & 3.59 & Salpeter \\
Leo 2a & 0.05 & 2 & 1 & 0 & 7 & 2.12 & 3.67 & Salpeter \\
Leo 2b & 0.30 & 8 & 1 & 0 & 7 & 2.69 & 1.54 & Salpeter \\
Leo 2c & 1.50 & 11 & 1 & 0 & 7 & 7.03 & 2.68 & Salpeter \\
\hline
\end{tabular}

The SFR $\psi(t)$ has a simple form and is given by

$\psi(t)=v G(t)$,

where $v$ is the efficiency of star formation, namely the inverse of the typical timescale for star formation, and is expressed in $\mathrm{Gyr}^{-1}$.

In order to get the best agreement with the abundance ratios and the metallicity distribution, $w_{i}$ and $v$ are varied in each galaxy following the procedure of LM03. The star formation is not halted even after the onset of the galactic wind, but proceeds at a lower rate, since a large fraction of the gas $(\sim 10 \%$ of the total lumionius mass) is carried out of the galaxy. The details of the star formation are given by the star formation history of each individual galaxy as inferred by observed color-magnitude diagrams (CMD) in Dolphin et al. (2005, see Table 1 for more details).

The rate of gas infall is defined as

$\left(\dot{G}_{i}\right)_{\mathrm{inf}}=A \mathrm{e}^{-t / \tau}$

with $A$ a suitable constant and $\tau$ the infall timescale which is assumed to be $0.5 \mathrm{Gyr}$. The constant $A$ is assumed to be the same as in the solar vicinity and represents the fraction of binary systems in the IMF which are the progenitors of SNe Ia.

The rate of gas loss via galactic winds for each element $i$ is assumed to be proportional to the star formation rate at the time $t$

$\left(\dot{G}_{i}\right)_{\text {out }}=w_{i} \psi(t)$,

where $w_{i}$ is a free parameter describing the efficiency of the galactic wind, which is the same for all heavy elements.

\section{Results}

The predictions of the chemical evolution models for Leo 1 and Leo 2 were compared with the available observational data of these two galaxies. In particular, the evolution of several $[\alpha / \mathrm{Fe}]$ ratios $(\mathrm{Ca}, \mathrm{Si}, \mathrm{O}$ and $\mathrm{Mg}$ ), [s-process/Fe], [r-process/Fe], and the stellar metallicity distribution were used as main constraints. We considered two stellar metallicity distributions, one as a function of $[\mathrm{Fe} / \mathrm{H}]$ and another as a function of $[\mathrm{Ca} / \mathrm{H}]$. In fact, the observed $[\mathrm{Fe} / \mathrm{H}]$ were derived from the $\mathrm{Ca}$ triplet lines and may not trace the effective iron abundance (Lanfranchi et al. $2006-$ LMC06b; Bosler et al. 2004, 2007), as predicted in the models. The abundance of $\mathrm{Ca}$ on the other hand was inferred through the atmospheric abundance analysis of neutral calcium and was corrected for non-LTE effects

The SF timescale in the galaxy and the nucleosynthesis of several chemical elements can be analyzed through particular abundance ratios due to the difference in the formation and injection of these elements into the ISM. The $[\alpha / \mathrm{Fe}]$ ratio for instance can be used as a "chemical clock" since $\alpha$-elements are produced mainly in SNe II explosions in short timescales, whereas explosions of SNe Ia are the main site for the production of $\mathrm{Fe}$ peak elements on a much longer timescale. Consequently, a long SF timescale and an older age are characterized by low $[\alpha / \mathrm{Fe}]$ values whereas a high $[\alpha / \mathrm{Fe}]$ ratio is the result of a short $\mathrm{SF}$ timescale and a younger age. Abundance ratios between neutron process elements are also used to impose constraints in the SF timescale and in the formation of the elements when r-process elements are compared to s-process, since the main site of the production of these two types of elements are quite different: the main source of r-process elements are SNe II explosions, whereas low and intermediate mass stars (LIMS) are believed to be the main site for the production of s-process elements (Woosley et al. 1994; Gallino et al. 1998; Freiburghaus et al. 1999; Busso et al. 2001; Wanajo et al. 2003).

Besides abundance ratios, SMDs can be used as powerful tools to investigate the chemical evolution of the galaxy. The general shape of the distribution along with its details can reveal how the stars formed and evolved in a galaxy and also impose constraints on the physical process acting on it. An absence of a metal-poor tail can be the consequence of a pre-enriched gas from which the stars formed, or the result of a slow infall of gas. Besides that, the position of the peak of the distribution is related to the SFR (if it is low or high) and to the duration of the SF episode (or episodes) as well as to the assumed IMF, and the metal-rich tail can impose constraints on the occurrence of galactic winds and in their efficiency in removing the gas of the system.

By comparing the predictions of our model with key abundance ratios and the SMD observed in Leo1 and Leo 2 we hope to improve the understanding of the chemical evolution of these systems. In particular, we suggest a scenario for the formation and evolution of these galaxies by describing some of its main parameters such as the star formation history, the SFR, the IMF, the occurrence of galactic winds and how efficiently they removed the gas out of the galaxy, the epoch the SF was halted, and others.

In this work, however, the strongest observational constrains on the model's parameters come from the stellar metallicity distribution due to the particularities of the adopted data sample, specially the ones concerning the abundance ratios (low number of stars in Leo 1 and large dispersion in Leo 2). The abundance ratios are used as a first filter, which allows us to pre-define the IMF, the SFH and a range (a broad one in this case) of acceptable values for the star formation and galactic wind efficiencies. A best model is then defined with specific values for these two parameters (within the range of values) and for the infall timescale based on the special features of the stellar metallicity distributions of each galaxy.

\subsection{Leo 1}

Leo 1 is one of the most distant $\mathrm{dSph}$ galaxies in the Local Group believed to be associated to the Milky Way. A few studies 
concerning the chemical evolution of Leo 1 have been published in the last few years, most of them focused in the star formation history, chemical abundances and the stellar metallicity distribution (Gallart et al. 1999; Held et al. 2000; Tolstoy et al. 2003; Shetrone et al. 2003; Dolphin et al. 2005; Bosler et al. 2007; Koch et al. 2007b). The main stellar population of Leo 1 seems to be very old (Held et al. 2001; Tolstoy et al. 2003), as in many other $\mathrm{dSph}$, but there are also hints of a younger population which would have been formed between 1 to 7 Gyr ago (Gallart et al. 1999; Dolphin et al. 2005). The differences in the star formation histories (SFH) and their consequences in the evolution of the galaxy can be addressed by detailed chemical evolution studies, which take into account other processes like the inflow and outflow of gas (LMC06b).

The SMD and abundance ratios can also provide some clues to the formation and evolution of Leo1. Unfortunately, there are only a few stars with spectroscopic abundance measurementsfor this galaxy (Shetrone et al. 2003), which by themselves do not allow one to draw any firm conclusion about the evolution of the galaxy. However, when these abundances are analyzed together with the SMD a definite scenario emerges. As mentioned previously, since the number of stars with determined abundance ratios is small, the stellar metallicity distribution turns out to be the strongest constraint on the models. The SMD of Leo 1 was recently studied in detail by Gullieuszik et al. (2009), Koch et al. (2007b), and Bosler et al. (2007). In all cases, the CaT method was used to derive the $[\mathrm{Fe} / \mathrm{H}]$, and the mean metallicity found was between $[\mathrm{Fe} / \mathrm{H}]=-1.31$ dex (Koch et al. 2007b) and -1.40 dex (Gullieuszik et al. 2009), with an intermediate value of $[\mathrm{Fe} / \mathrm{H}]=-1.34$ dex found by Bosler et al. (2007). Bosler et al. (2007) also derived $[\mathrm{Ca} / \mathrm{H}]$ and found a mean value of -1.34 dex. The SMD is well described by Bosler et al. (2007) and Koch et al. (2007b) by a Gaussian function with a full range in metallicity of approximately 1.0 dex.

All these features together lead to a scenario in which the $\mathrm{dSph}$ galaxy Leo 1 is characterized by a fast enrichment from an initial generation of stars followed by a loss of metals by efficient galactic winds, as suggested by the results of simple closed-box models (Koch et al. 2007b; Gullieuszik et al. 2009). Besides that, Gullieuszik et al. (2009) suggested also that the first generation of stars in the galaxy was formed by pre-enriched gas to account for the apparent lack of metal-poor stars in the stellar metallicity distribution, whereas Koch et al. (2007b) claim that the galaxy should not have been affected by Galactic tides, as it represents an isolated system.

By means of a detailed chemical evolution model with inflow and outflow of gas compared to the observed data, we investigated the proposed scenarios for the evolution of Leo 1.

\subsubsection{Abundance ratios}

First we compare the predictions for the Leo 1 model to the observed abundance ratios of several species like $[\alpha / \mathrm{Fe}]$ and $[\mathrm{Ba}$, $\mathrm{Eu} / \mathrm{Fe}$ (Fig. 1). Even though the number of points is low we are able to gain some insight into the SF and galactic wind efficiencies by defining the upper and lower limits of a range of values for these two parameters. This range of values allows the model to account for the majority of points (models Leo1a and Leo1c - Table 1). A low SF efficiency $\left(v=0.15-1.7 \mathrm{Gyr}^{-1}\right)$ gives rise to values of $[\alpha / \mathrm{Fe}]$ above solar and very low $[\mathrm{s}, \mathrm{r} / \mathrm{Fe}]$ at low metallicities $([\mathrm{Fe} / \mathrm{H}]<-3.0 \mathrm{dex})$. As the metallicity increases, $[\alpha / \mathrm{Fe}]$ decreases slowly, whereas $[\mathrm{Ba}, \mathrm{Eu} / \mathrm{Fe}]$ increases fast at $[\mathrm{Fe} / \mathrm{H}] \sim-3.0$ dex and then reaches some kind of a plateau around solar values. The decrease in $[\alpha / \mathrm{Fe}]$ is a consequence of

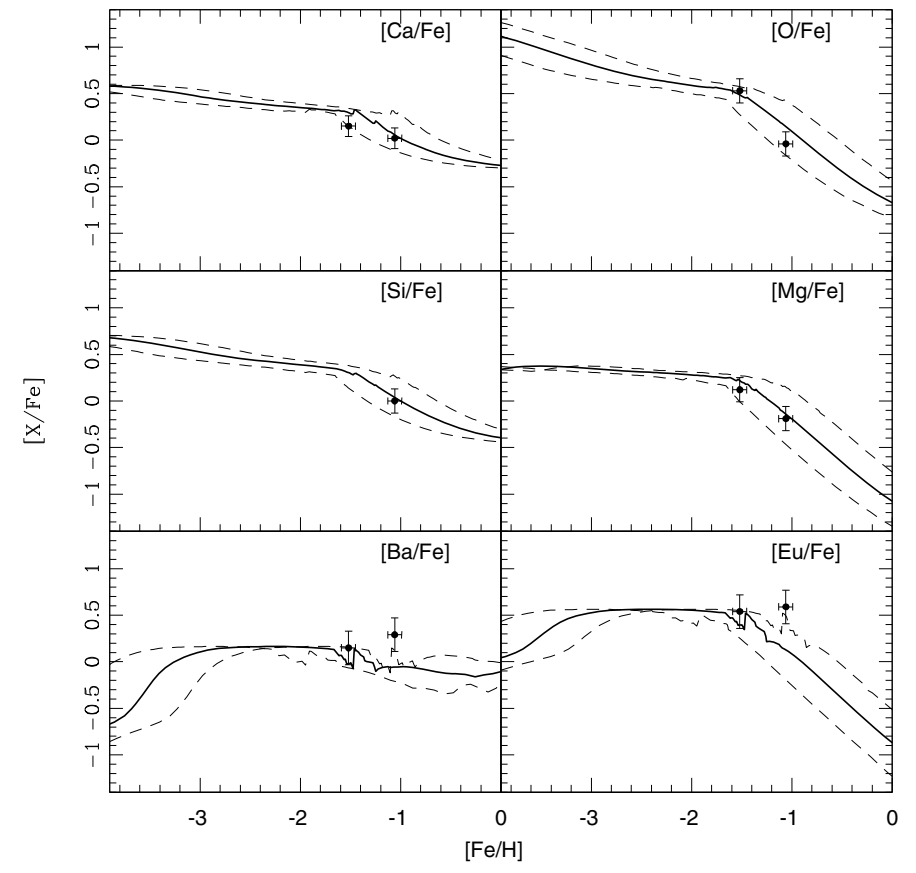

Fig. 1. $[\mathrm{X} / \mathrm{Fe}]$ vs. $[\mathrm{Fe} / \mathrm{H}]$ observed in the dSph galaxy Leo 1 compared to the predictions of the best model (solid line). The dashed lines represent the upper and lower limits of the predictions.

the slow injection of Fe into the ISM by SNe Ia, which starts to explode from several Myr to several Gyr after the first explosions of SNe II (responsible for the main component of alpha and rproduced elements). In [ $\mathrm{Ba}, \mathrm{Eu} / \mathrm{Fe}]$, the increase is a result of the production by r-process of these elements in SNe II explosions originating from stars with masses inferior to $M=30 M_{\odot}$.

From intermediate to high metallicities $([\mathrm{Fe} / \mathrm{H}]>-1.5 \mathrm{dex})$ one can notice a steep decline in $[\alpha / \mathrm{Fe}]$ and $[\mathrm{Eu} / \mathrm{Fe}]$ and a smoother one for $[\mathrm{Ba} / \mathrm{Fe}]$ (the oscilations seen around $[\mathrm{Fe} / \mathrm{H}]=-$ 1.5 dex in the bottom panels are caused by small numerical fluctuations in the code). This sudden change in the abundance patterns is a consequence of the occurrence of a strong galactic wind $\left(w_{i}=6-10\right)$ when the thermal energy of the galaxy equates or exceeds its binding energy. The main factor that affects the thermal energy of the gas is the energy injected into the medium by $\mathrm{SNe}$ explosions, which gives rise to a wind short after the first generation of SNe Ia (depending on the SF efficiency). With the onset of the galactic wind the gas content of the ISM starts to be removed from the galaxy and therefore the SFR starts to decrease. The number of new stars formed after the onset of the wind is much lower, as is the amount of alpha elements and Eu (r-processed element) injected into the ISM. The main source of these elements (SNe II explosions) originates from massive stars which evolve and die fast (tens to hundred Myr), enriching the medium soon after they are formed. Iron on the other hand, is produced mainly in SNe Ia during a much longer time. Because of that the ISM continues to be enriched in Fe even after the onset of the wind by the stars which were born before the wind started and died only afterwards.

This sudden interruption in the production of Eu and alpha elements combined with the injection of Fe in the ISM by SN Ia leads to the sharp decrease in the predictions of the model, similar to what is observed in the data. Barium, unlike Eu and the alpha elements, has two main different productions - from the $s$ and from the $r$ process. As mentioned before, SNe II explosions are believed to be the site for the production of r-processed 
elements, but the s-process is claimed to take place in low and intermediate mass stars (LIMS) (Busso et al. 2001; Woosley et al. 1994). Consequently, the s-process component of Ba continues to be injected into the ISM for a few Gyr (the timescale of LIMS), which causes a smoother decline in $[\mathrm{Ba} / \mathrm{Fe}]$.

It is evident from all this that the efficiency with which the galactic wind removes the gas of the galaxy is crucial in determining the pattern of the abundance ratios, especially at high metallicities where the galactic wind plays a major role. If the gas removal is not efficient enough $\left(w_{i} \sim 1\right.$, for instance), the decrease in the predictions does not reach the lowest values observed, in particular for $[\alpha / \mathrm{Fe}]$. These can only be reproduced by a wind with a rate several times higher than the $S F R-w_{i}=6-$ 10 in the case of Leo 1. Besides that, the low SF efficiency $\left(v=0.15-1.7 \mathrm{Gyr}^{-1}\right)$ explains the patterns observed. If $v$ is higher than that, the predictions of all abundance ratios at low metallicities will be also higher, the wind will develop at higher $[\mathrm{Fe} / \mathrm{H}]$, shifting the decrease towards the right in the plots, and the agreement between predictions and data will be lost. The stellar metallicity distribution is also strongly affected by these parameters as we will discuss below.

\subsubsection{The stellar metallicity distribution}

The general shape, the low and high-metallicity tails, and the position of the peak of the stellar metallicity distribution are strongly affected by the choice of parameters of the chemical evolution model. By comparing the predictions of the model with the observed data we are able to verify and in particular narrow the range of values for the main parameters (specially the wind and SF efficiencies) already selected from the comparison with the abundance ratios. The SMD, in this sense, allows one to define a best model with specific values for the star formation and galactic wind efficiencies within the range of values determined by the comparison with the abundance ratios and to test the previous choice of the IMF and the infall timescale. The upper and lower values previously determined could be interpreted as limits of the model's predictions.

The predictions of the best model for Leo 1 (Leolb - straight line) compared to the observed SMDs (long dashed line) are show in Figs. 2 and 3 (as a function of $[\mathrm{Fe} / \mathrm{H}]$ and $[\mathrm{Ca} / \mathrm{H}]$, respectively) together wiht the upper and lower models. In both cases the general shape of the distribution, the position of the peak and the low metallicity tail can be well reproduced by the predictions of the best model (Leo1b). It adopts in particular values near the lower limit for the efficiency of the star formation $\left(v=0.6 \mathrm{Gyr}^{-1}\right)$, whereas for the galactic wind efficiency the best value is near the higher limit $\left(w_{i}=9\right)$.

At low metallicities, the low number of stars observed is very well reproduced by the best model in both cases (the fit for $[\mathrm{Ca} / \mathrm{H}]$ is excellent) without the need to adopt a pre-enriched gas to form the galaxy, as suggested by Gullieuszik et al. (2009). The very low SF efficiency and the infall timescale are the main factors leading to this scenario. The gradual formation of the galaxy ensures that few metal poor stars form since there is little gas at the very beginning and SNeII rapidly pollute the infalling gas.

The position of the peaks in both distributions (as a function of $[\mathrm{Fe} / \mathrm{H}]$ and of $[\mathrm{Ca} / \mathrm{H}]$ ) at metallicities much lower than the one of the SMD from the solar neighborhood $([\mathrm{Fe} / \mathrm{H}] \sim-1.4 \mathrm{dex}$ and $[\mathrm{Fe} / \mathrm{H}] \sim 0.0$ dex, respectively) is a consequence of the low SFR coupled with the occurrence of a galactic wind. Of great importance in this case is the efficiency of the galactic wind, i.e. the rate at which the gas is removed from the galaxy. With a large

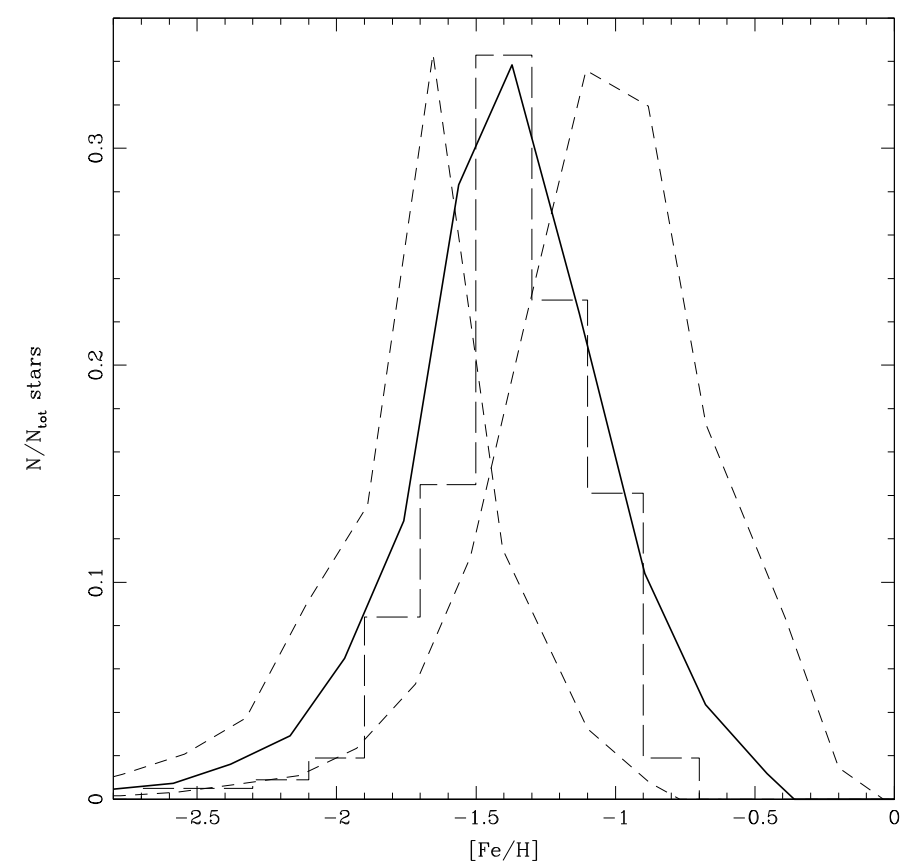

Fig. 2. The stellar metallicity distribution as a function of $[\mathrm{Fe} / \mathrm{H}]$ observed in the dSph galaxy Leo 1 (long dashed line) compared to the predictions of the best model (solid line). The short dashed lines represent the upper and lower limits of the predictions.

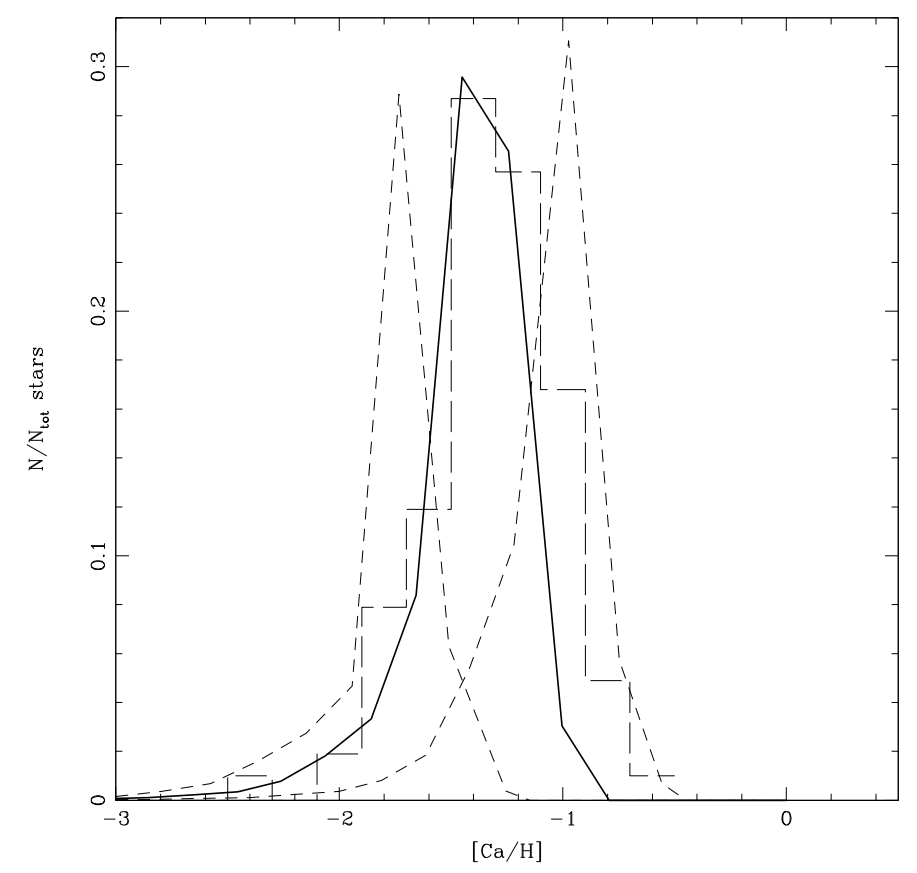

Fig. 3. The stellar metallicity distribution as a function of $[\mathrm{Ca} / \mathrm{H}]$ observed in the dSph galaxy Leo 1 (long dashed line) compared to the predictions of the best model (solid line). The short dashed lines represent the upper and lower limits of the predictions.

efficiency $\left(w_{i}=9\right)$, the wind removes a large fraction $(\sim 10 \%)$ of the gas content of the galaxy as soon as it starts. As a consequence, the number of stars which are formed with metallicities higher than the gas metallicity at the time of the beginning of the wind is reduced dramatically.

The high wind efficiency also defines the shape of the metalrich tail of the distribution. As can be seen in the data, the 
more metal-rich stars do not reach metallicities as high as $[\mathrm{Fe} / \mathrm{H}] \sim-0.5 \mathrm{dex}$, and the decline in the SMD is very sharp after the peak. This is normally explained as due to the termination of the SF. In the models the SF is not halted, but substantially decreased after the wind starts to sweep away the ISM gas from the galaxy. In the model predictions, however, a difference can be noticed between the two distributions (Figs. 2 and 3). The SMD as a function of $[\mathrm{Ca} / \mathrm{H}]$ exhibits a very sharp decrease at high metallicities, whereas the one as a function of $[\mathrm{Fe} / \mathrm{H}]$ is more symmetric. This difference can be explained by taking into account the different sites of the production of $\mathrm{Fe}$ and $\mathrm{Ca}$. Both elements are produced partially in SNe II and partially in SNe Ia, but the fraction of each element that is produced in each type of supernovae is very different. $\mathrm{Ca}$ is mainly produced by SNe II on a short timescale, whereas the main fraction of Fe is produced on a much longer timescale, by SNe Ia. Even after the SF has become very low (the wind is carrying gas away), a considerable fraction of $\mathrm{Fe}$ is injected into the ISM contrary to $\mathrm{Ca}$, whose enrichment is almost stopped after the wind. Therefore the differences between the two observed SMDs could be attributed to this fact, but partly also to the procedure adopted to estimate the abundances of the two elements ( $\mathrm{Fe}$ and $\mathrm{Ca}$ ) and to the different data sets used to construct the two SMDs.

Since the abundance of $[\mathrm{Fe} / \mathrm{H}]$ is normally derived from $\mathrm{Ca}$ triplet lines and this procedure can lead to some discrepancies, especially when applied to extragalactic stars, Bosler et al. (2007) estimated also the abundance of $[\mathrm{Ca} / \mathrm{H}]$. These authors proposed a new procedure to estimate the metallicity of stars in local galaxies using $\mathrm{Ca}$ as an indicator to avoid the dependence of $[\mathrm{Fe} / \mathrm{H}]$ on $[\mathrm{Ca} / \mathrm{Fe}]$ built into the CaII calibrations. The $[\mathrm{Ca} / \mathrm{H}]$ values were determined from an atmospheric abundance analysis of neutral calcium and were corrected for non LTE effects. As the obtained $[\mathrm{Ca} / \mathrm{H}]$ does not strongly depend on the $[\mathrm{Ca} / \mathrm{Fe}]$, the stellar ages, and the SFH of the galaxy, Bosler et al. (2007) adopted this abundance as a tracer of the metallicity. Apart from these differences the observed data are not the same in the two plots. In the Ca plot we used only the data from Bosler et al. (2007) (the only work that estimated this abundance), whereas in the Fe plot we put together all the data from Bosler et al. (2007), Koch et al. (2007b) and Gullieuszik et al. (2009). In that sense, even though there could be errors in the determination of $[\mathrm{Fe} / \mathrm{H}]$, the set of data for this element is more substantial and perhaps not homogeneous. Considering all this and looking for a more complete picture we compared our predictions with both SMD.

Models $a$ and $c$ of Leo 1 predict SMDs with peaks at lower and higher $[\mathrm{Fe} / \mathrm{H}]$, respectively, compared to the observations, due mainly to the limiting values chosen for the SF efficiency. The SMDs exhibit also low and high-metallicity tails shifted to higher and lower metallicities with respect to the observed ones. Other than that, the different values adopted for the star formation and galactic wind efficiencies can help to explain small differences between the predictions of the best model and the observed data. In Fig. 2 the predicted SMD seems to be broader than the observed one, and this can be related to the adopted SF and wind efficiencies. By comparing the predictions of the best model with the model Leo1a (lower limits - curve on the left) one can notice that the latter shows a sharper distribution. On the other hand, model Leo1c (with higher values for the main parameters) exhibits a broader distribution compared to the best model. A more complete set of stars with measured abundance ratios could help to adjust these values and provide a better fit to the SMD.

\subsection{Leo 2}

Understanding the chemical enrichment history of the dwarf spheroidal galaxy Leo 2 (and of Leo 1) contributes to increase the knowledge of the processes which affect the evolution of this class of objects, since this is the second most distant known dwarf that is assumed to be orbiting our Galaxy (Bellazzini et al. 2005). If isolated dSphs exhibit similar chemical properties as the most nearby ones, it could imply that these properties in dSph are results mainly of their stellar and chemical evolution.

Leo 2 is a metal-poor system dominated by intermediate age populations (Dolphin et al. 2005; Bellazzini et al. 2005; Mighell \& Rich 1996). The SF occurred mainly at early epochs (until approximately 9 Gyr ago), but there are also hints of more recent SF (Dolphin et al. 2005). Its stellar metallicity distribution is characterized by an apparent lack of metal-poor stars, similar to other dSphs, with an average metallicity around $[\mathrm{Ca} / \mathrm{H}] \sim$ -1.65 dex (Bosler et al. 2007) and $[\mathrm{Fe} / \mathrm{H}] \sim-1.74$ dex (Koch et al. 2007a). The spread in metallicity also differs when different elements are adopted as metallicity tracers: for $[\mathrm{Ca} / \mathrm{H}]$ the metallicity ranges from $-2.61 \geq[\mathrm{Ca} / \mathrm{H}] \geq-0.59$ dex (Bosler et al. 2007 ), whereas it ranges from -2.4 to -1.08 dex when $[\mathrm{Fe} / \mathrm{H}]$ is considered (Koch et al. 2007a). In both cases, however, the general shape exhibits an asymmetric distribution, with a rapid and sharp falloff at the metal-rich tail, which is normally attributed to the sudden suppression of the SF and the loss of gas.

More recently, Shetrone et al. (2009) used previously published spectra to derive abundance ratios of $\mathrm{Mg}$ and $\mathrm{Ca}$ for almost 30 stars in Leo 2. Their results show a trend of a gradual decline of $[\mathrm{Mg} / \mathrm{Fe}]$ and $[\mathrm{Ca} / \mathrm{Fe}]$ with increasing metallicity in more metal-rich stars, similar to what is observed in nearby dSphs. Consequently, these authors suggested that this trend supports the hypothesis that the slow chemical enrichment of dSph does not depend on any interaction with the Milky Way galaxy, since this galaxy is located at a large galactocentric distance. A further analysis taking into account the flows of gas with a detailed chemical evolution model can help to shed light on this subject.

\subsubsection{Abundance ratios}

The predictions for $[\mathrm{Mg} / \mathrm{Fe}]$ and $[\mathrm{Ca} / \mathrm{Fe}]$ ratios from the Leo 2 models are compared to the observed data in Figs. 4 and 5, respectively. Contrary to the several chemical species estimated in only a low number of stars in Leo 1, in Leo 2 the abundances were inferred for a considerable number of stars (almost 30), but only for a few elements. $\mathrm{Mg}$ and $\mathrm{Ca}$ are enough, though, to represent the pattern on $[\alpha / \mathrm{Fe}]$, even though some differences exist in their production. $\mathrm{Mg}$ is almost totally produced in SNe II (similar to $\mathrm{O}$ ), whereas a fraction of $\mathrm{Ca}$ is also produced in $\mathrm{SNe} \mathrm{Ia}$ (similar to $\mathrm{Si}$ ). In fact, this difference is helpful to impose constraints in the chemical history of the galaxy and in the parameters of the models.

Both abundance ratios analyzed here are characterized by a very large dispersion which makes it difficult to establish a precise trend. The general features are very similar however: at low metallicities $([\mathrm{Fe} / \mathrm{H}] \sim-2.5 \mathrm{dex})$ they exhibit values roughly above solar $(\sim 0.5 \mathrm{dex})$, similar to what is observed in Leo 1 and in other dSphs. These values start decreasing very smoothly as the metallicity increases. This is consistent with a slow SF (with a low efficiency $-v=0.05-1.50 \mathrm{Gyr}^{-1}$ ) as the model predictions show. The evolutionary tracks provided by the models decrease from $[\mathrm{Mg} / \mathrm{Fe}] \sim 0.3$ to $\sim 0.2$ dex and from $[\mathrm{Ca} / \mathrm{Fe}] \sim 0.4$ to $\sim 0.3$ in the range $[\mathrm{Fe} / \mathrm{H}] \sim-2.5$ to -1.7 dex. Neither the upper nor 


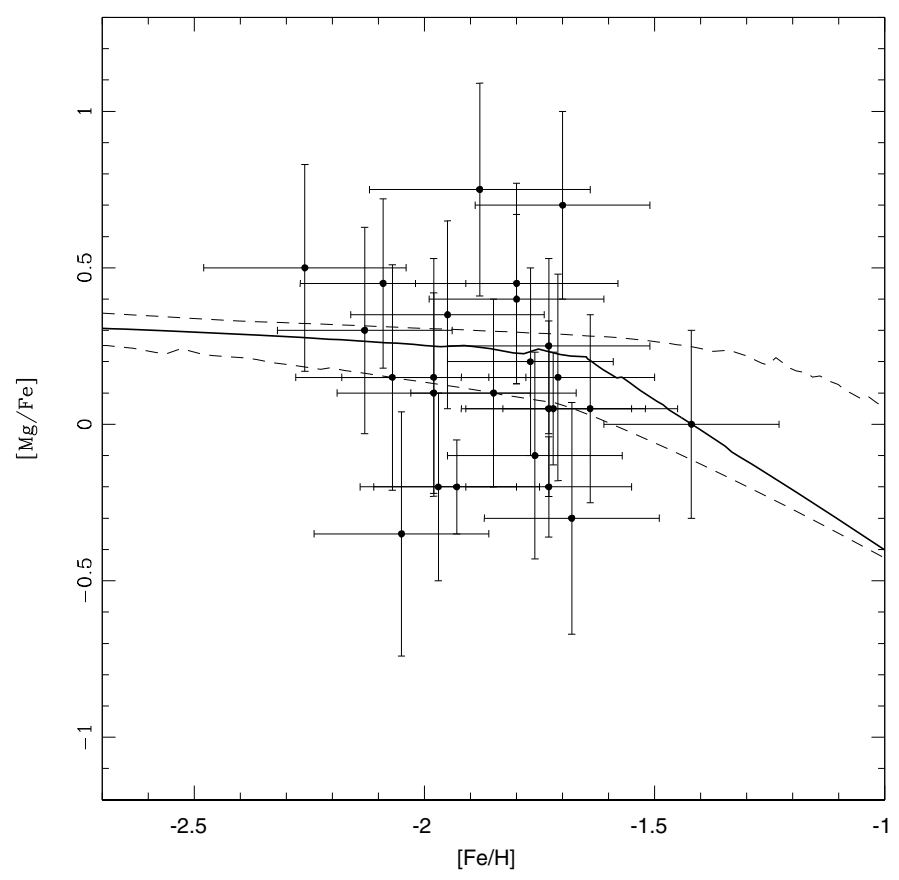

Fig. 4. $[\mathrm{Mg} / \mathrm{Fe}]$ vs. $[\mathrm{Fe} / \mathrm{H}]$ observed in the dSph galaxy Leo 2 compared to the predictions of the best model (solid line). The dashed lines represent the upper and lower limits of the predictions.

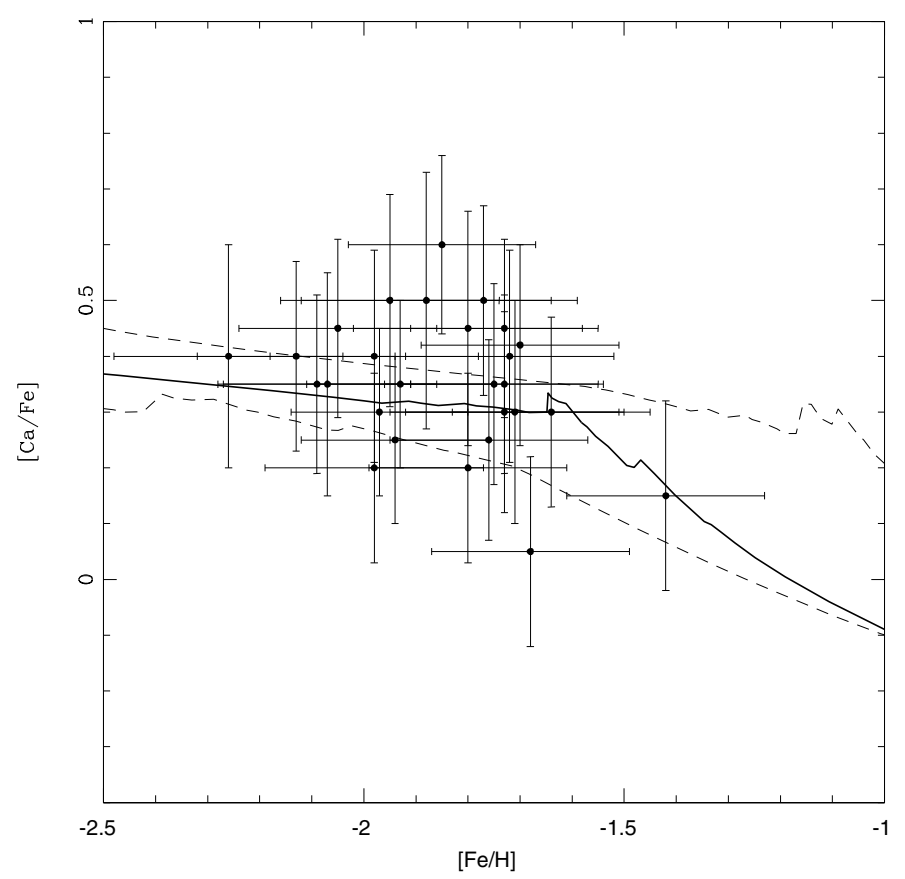

Fig. 5. $[\mathrm{Ca} / \mathrm{Fe}]$ vs. $[\mathrm{Fe} / \mathrm{H}]$ observed in the dSph galaxy Leo 2 compared to the predictions of the best model (solid line). The dashed lines represent the upper and lower limits of the predictions.

the lower models (Leo2a and Leo2c) can account for all the data due to the large dispersion, but all three tracks show a similar behavior of a decreasing abundance ratio with increasing metallicity. As explained in the previous section, the slow change in the values of these abundance ratios is caused by the low SFR and consequent slow injection of $\mathrm{Fe}$ produced by $\mathrm{SNe}$ Ia into the ISM. From intermediate to high metallicities $([\mathrm{Fe} / \mathrm{H}]>-$ $1.7 \mathrm{dex})$ there is a break in the evolutionary tracks and a subsequent steep decline following the trend of the observed data

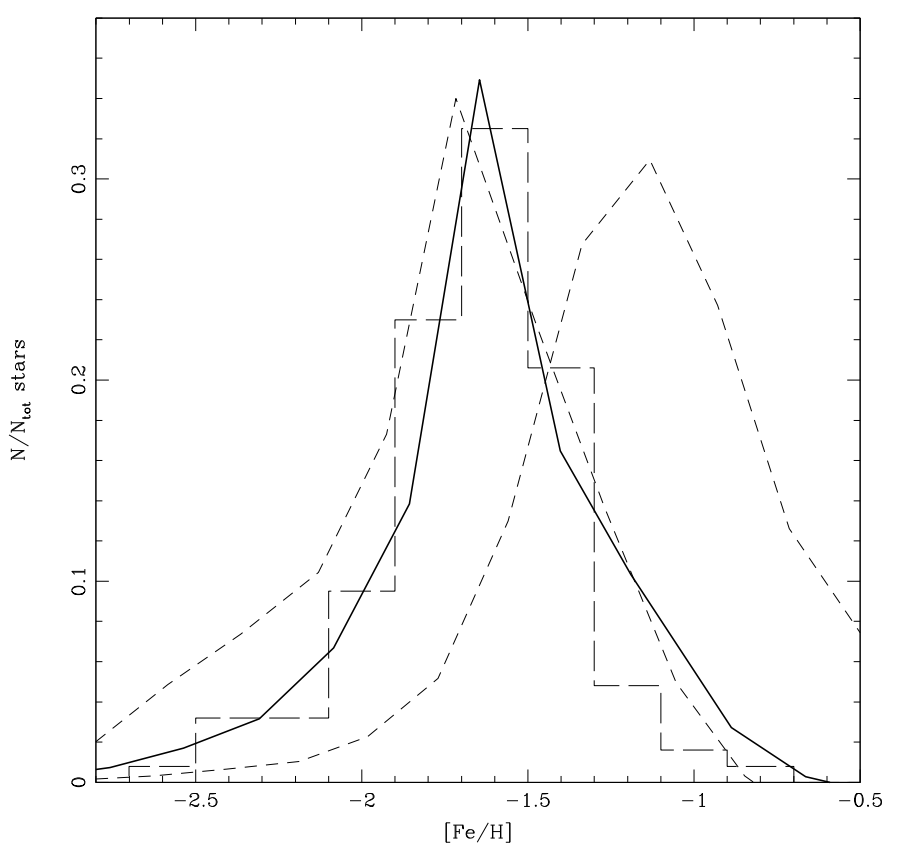

Fig. 6. The stellar metallicity distribution as a function of $[\mathrm{Fe} / \mathrm{H}] \mathrm{ob}-$ served in the dSph galaxy Leo 2 (long dashed line) compared to the predictions of the best model (solid line). The short dashed lines represent the upper and lower limits of the predictions.

(in this case also the oscilations seen in the predictons around $[\mathrm{Fe} / \mathrm{H}]=-1.6$ dex are caused by small numerical fluctuations of the code). This break is caused mainly by the effects of the galactic winds on the SF. An intense galactic wind $\left(w_{i}=2-11,2-11\right.$ times the SFR) carries away a large fraction of the gas content of the galaxy as soon as it starts, which substantially decreases the formation of new stars from that moment on. With the suppression of the SF, alpha elements are no longer injected into the ISM due to the short timescale of the stars responsible for its main bulk (around $30 \mathrm{Myr}$ for a $8 M_{\odot}$ star). Iron peak elements on the other hand continue to enrich the medium for a longer time (up to several billion years). As a consequence, the $[\alpha / \mathrm{Fe}]$ ratios decrease sharply after the onset of the wind as can be seen in the observations and in the predictions of the models.

\subsubsection{The stellar metallicity distribution}

The comparisons between the SMDs and the predictions of the models are shown in Figs. 6 and 7 (as a function of $[\mathrm{Fe} / \mathrm{H}]$ and $[\mathrm{Ca} / \mathrm{H}]$, respectively). Similar to Leo 1 , the SMD helps to define a best model with specific values for the efficiencies of the $\mathrm{SF}$ and galactic winds $\left(v=0.3 \mathrm{Gyr}^{-1}\right.$ and $w_{i}=8-$ Leo2b). For Leo 2, however, models $a$ and $c$ predict SMDs very different from the observed one. This difference could originate in the large dispersion observed in the abundance ratios (the constraint for the upper and lower models). In both cases the general agreement between the best model (Leo2b - solid thick line) and the observations is very good, with minor differences between the comparisons for each element (as in the case of Leo 1). At the poor-metal tail of the distributions, the number of stars predicted by the best model fits the observations very well when Fe is used as a metallicity tracer, whereas there seems to be a small underproduction of stars when $[\mathrm{Ca} / \mathrm{H}]$ is adopted. This difference is negligible though and could be a consequence of the metallicity calibration adopted in the different works. Similar to the case of Leo 1, the low number of metal-poor stars in the 


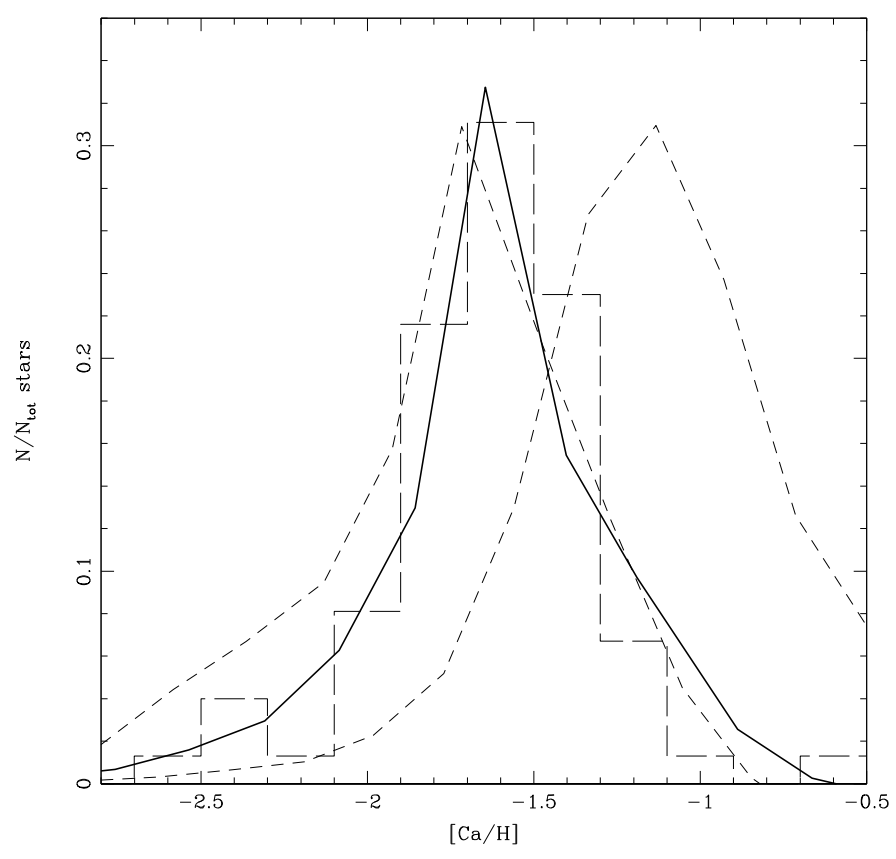

Fig. 7. The stellar metallicity distribution as a function of $[\mathrm{Ca} / \mathrm{H}]$ observed in the dSph galaxy Leo 2 (dashed line) compared to the predictions of the best model (long solid line). The short dashed lines represent the upper and lower limits of the predictions.

models and observations can be attributed to the very low SFR and the timescale of the infall, without the need to invoke an infall of pre-enriched gas.

In both cases $([\mathrm{Fe} / \mathrm{H}]$ and $[\mathrm{Ca} / \mathrm{H}])$ the observed and predicted metallicity peaks of the main stellar population lie between $[\mathrm{Fe} / \mathrm{H}]($ or $[\mathrm{Ca} / \mathrm{H}]) \sim-1.7-1.5 \mathrm{dex}$. As mentioned in the case of Leo 1, the metallicity of the peak of the distribution is determined by the low SF efficiency $\left(v=0.3 \mathrm{Gyr}^{-1}\right)$ and the high galactic wind efficiency $\left(w_{i}=8\right)$.

The effects of the wind on the SF can also be noticed in the metal-rich tail of the distribution. In the observed SMDs as a function of $[\mathrm{Fe} / \mathrm{H}]$ and $[\mathrm{Ca} / \mathrm{H}]$ a steep decline can be seen after the peak, due to the decrease in the number of stars formed. This decrease is normally attributed to the loss of the gas content that fuels the SF. In our scenario the gas is lost through intense galactic winds, triggered by SNe explosions. The predictions of the models fit the observations nicely with a small overprediction on the number of stars by the best model (Leo2b - solid line). This discrepancy is mainly related to the efficiency of the galactic wind adopted in the best model. A higher value would decrease the SFR even more, decreasing also the number of stars with high $[\mathrm{Fe} / \mathrm{H}]$. Apart from that, one can see distinct differences in the metal-rich tails of each distribution: for the $[\mathrm{Ca} / \mathrm{H}]$ distribution the decline is sharp and fits the observations very well (with the exception of one star with $[\mathrm{Ca} / \mathrm{H}] \sim-0.5 \mathrm{dex}$ ); the metal-rich tail of the $[\mathrm{Fe} / \mathrm{H}]$ distribution seems to predict a small overabundance of stars. The slightly high number of stars with a high $[\mathrm{Fe} / \mathrm{H}]$ content can be explained by taking into account the main site of the production of this element and the long timescale for its injection in the ISM, the reason for which can be found in the different nucleosynthesis sites of $\mathrm{Fe}$ and $\mathrm{Ca}$, as already discussed for Leo I. In the observed data, as also explained before, the differences could be partly the result of the methods adopted in different works to derive the abundances of $\mathrm{Ca}$ and $\mathrm{Fe}$ and to the fact that in the $[\mathrm{Ca} / \mathrm{H}]$ distribution only the data of Bosler et al. (2007) are used, at variance with the SMD as a function of $[\mathrm{Fe} / \mathrm{H}]$, which is constructed with all data together. Therefore it is not recommended to compare the two observed distributions, since it could confuse the interpretation. We decided to compared them both with observations to have a more complete picture of the chemical evolution of Leo 2, but kept in mind that the two should not be regard as the same.

\section{Discussion and conclusions}

We investigated the chemical evolutionary history of the dSph galaxies Leo 1 and Leo 2 by means of predictions from detailed chemical evolution models compared to observations. The stellar metallicity distributions of these galaxies and several abundance ratios were used as constraints to define the values for the main parameters of the models, like SF and galactic wind efficiencies. The SFHs were taken from results of color-magnitude diagrams, which indicate extended SF episodes which occur at early epochs, but also with the presence of intermediate stellar populations. Leo 1 is characterized by an intense episode occurring from 14 to 9 Gyr ago and a subsequent less intense SF, which lasts until approximately 3 Gyr ago, whereas Leo 2 formed stars also at early epochs but stopped 6-7 Gyr ago.

In our scenario, these galaxies formed from an initial collapse of pristine gas until a mass of $\sim 10^{8} M_{\odot}$ was reached. A SF with a very low efficiency $\left(v=0.6 \mathrm{Gyr}^{-1}\right.$ and $v=0.3 \mathrm{Gyr}^{-1}$ for Leo 1 and Leo 2 best models, respectively) takes place, and the galaxies start evolving slowly. The slow SF and the infall timescale give rise to a low number of metal-poor stars in agreement with the observations. Stars are forming slowly in time, but the overall metallicity increases relatively fast with the most massive stars exploding and injecting metal into the ISM on a very short timescale (few Myr for a $M>8 M_{\odot}$ ). After $t M_{\text {tot }}$ $\left(10^{6} M_{\mathrm{o}}\right)$ the first generation of stars, the ISM is enriched enough that the newborn stars contain already a considerable amount of metals. The SF proceeds for several Myr to a few Gyr when the majority of stars are formed. During this early epoch the $[\alpha / \mathrm{Fe}]$ ratios are characterized by values above solar, which decrease smoothly as a function of metallicity due to the slow enrichment of Fe by the first SNe Ia. As SNe continue to explode, energy is being released, and when the thermal energy accumulated in the ISM becomes equal or higher than the binding energy of the galaxy, an intense wind occurs. Almost all the evolution of the galaxy is defined until this moment, before the wind (see also Calura et al. 2008).

When the wind (with a efficiency $w_{i}=9$ for Leo 1 and $w_{i}=8$ for Leo 2) starts, a large fraction of the gas is lost from the medium and, consequently, the SF decreases substantially. The decline of the SFR drastically changes the pattern of the abundance ratios and defines the stellar metallicity distribution (in particular, the position of the peak and the metal-rich tail). There is a sharp decrease in the $[\alpha / \mathrm{Fe}]$ and $[r / \mathrm{Fe}]$ ratios since the production of both the $\alpha$ and r-process elements in $\mathrm{SNe}$ II is almost halted, whereas the enrichment of Fe continues for several Gyr (the timescale of the progenitors of SNe Ia). In the case of $[\mathrm{Ba} / \mathrm{Fe}]$ the values do not decline considerably because $\mathrm{Ba}$ is mainly produced by the s-process in LIMS which enrich the ISM for a long time even after the wind. The drop in the SFR also changes the SMD. The number of stars formed with metallicities higher than that of the ISM when the wind develops reduces drastically, so there is a sharp fall-off in the metal-rich tail of the distributions, and the peak in metallicity of the main population of stars is at a low value $([\mathrm{Fe} / \mathrm{H}] \sim-1.4 \mathrm{dex}$ and $-1.6 \mathrm{dex}$ for Leo 1 and Leo 2, respectively). The SF then continues, but at a lower rate, until it is negligible. 
Table 2. Models without galactic winds for the dSph galaxies Leo 1 and Leo 2.

\begin{tabular}{lcccccccc}
\hline \hline Galaxy & $v\left(\mathrm{Gyr}^{-1}\right)$ & $w_{i}$ & $n$ & $t(\mathrm{Gyr})$ & $d(\mathrm{Gyr})$ & $M_{\mathrm{tot}}\left(10^{7} M_{\mathrm{o}}\right)$ & $M_{\text {gas }}\left(10^{4} M_{\mathrm{o}}\right)$ & $I M F$ \\
\hline Leo1-nw1 & 0.60 & - & 2 & $0 / 5$ & $5 / 7$ & 5.26 & 38.1 & Salpeter \\
Leo1-nw2 & 0.30 & - & 2 & $0 / 5$ & $0.6 / 0.4$ & 5.34 & 43.5 & Salpeter \\
Leo2-nw1 & 0.30 & - & 1 & 0 & 7 & 30.6 & 14.0 & Salpeter \\
Leo2-nw2 & 0.15 & - & 1 & 0 & 0.9 & 2.00 & 43.7 & Salpeter \\
\hline
\end{tabular}

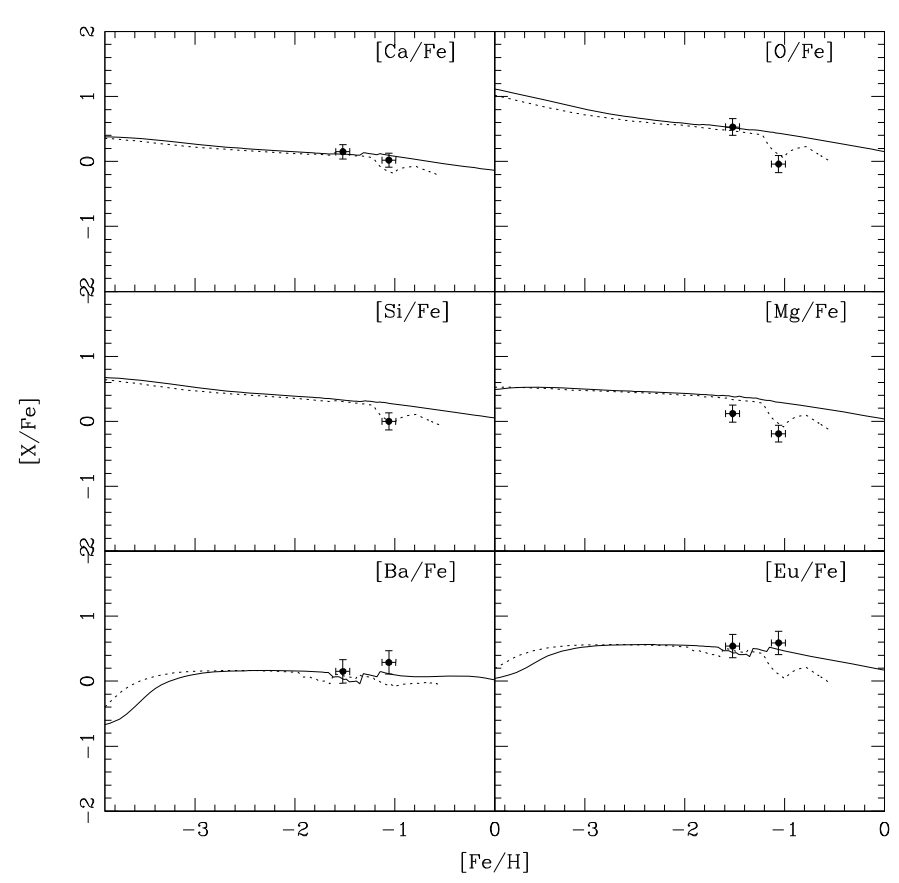

Fig. 8. $[\mathrm{X} / \mathrm{Fe}]$ vs. $[\mathrm{Fe} / \mathrm{H}]$ observed in the dSph galaxy Leo 1 compared to the predictions of the chemical evolution models with no galactic winds (Leo1-nw1: solid line, Leo1-nw2: dotted line).

It has been claimed though that dynamical interactions could remove preferentially the most metal-poor stars of dwarf galaxies (as it has been shown for Sagittarius - Monaco et al. 2007; Chou et al. 2007), thus modifying the observed present-day SMD. This point, if it were present in the dSph galaxies Leo 1 and Leo2, would imply a higher fraction of metal-poor stars and broader SMDs. Both features would require modifications in the main parameters of the models, in particular the SF and wind efficiency and the infall timescale. As explained earlier, a broader SMD can be achieved with slightly higher values for the efficiencies, whereas a higher fraction of metal-poor stars would probably mean a shorter infall timescale.

Nevertheless, the general trend observed in the $[\alpha / \mathrm{Fe}]$ ratios and the shape of the SMDs can be very well explained by a low SFR and the occurrence of a very intense galactic wind for both galaxies. Even though we can consider the loss of gas by winds as a reasonable physical explanation, a question should be raised. Could the observations also be explained without evoking loss of gas? As demonstrated in LMC07 and LM08 both the typical decrease in the abundance ratios at high metallicities and the shape of the stellar metallicity distributions observed in dSph galaxies are very difficult to reconcile if no gas loss is assumed. The steep decline in $[\alpha / \mathrm{Fe}]$ and $[\mathrm{Ba}, \mathrm{Eu} / \mathrm{Fe}]$ cannot be reproduced without the decrease in the SFR. As shown in LMC07 for Sagittarius, a model with no wind predicts higher values for these abundance ratios at high metallicities, well above the observed data. In Figs. 8 to 11 we show the predictions from models with the

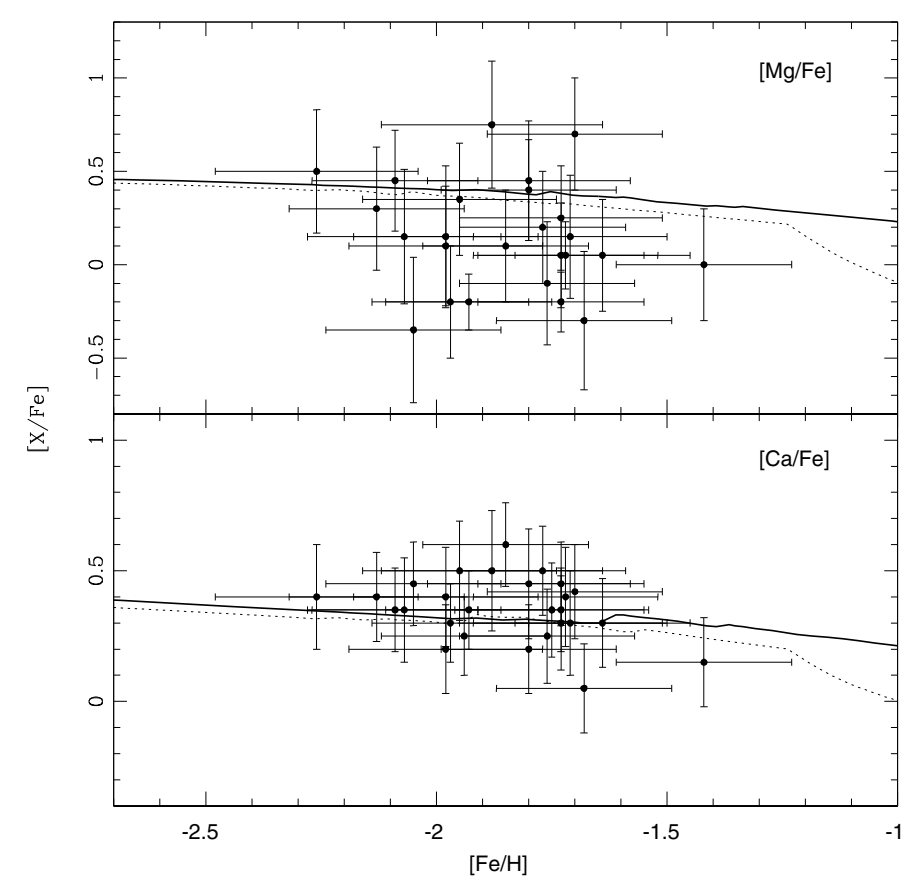

Fig. 9. $[\mathrm{Ca}, \mathrm{Mg} / \mathrm{Fe}]$ vs. $[\mathrm{Fe} / \mathrm{H}]$ observed in the dSph galaxy Leo 2 compared to the predictions of the chemical evolution models with no galactic winds (Leo2-nw1: solid line, Leo2-nw2: dotted line).

same parameters as the best models of Leo 1 and Leo 2, but with no galactic winds (models Leo1-nw1 and Leo2-nw1 - Table 2) compared to the observations of $[\alpha / \mathrm{Fe}]$ ratios. As we demonstrated in the case of Sagittarius, the decrease in the abundance ratios is not sharp enough to reproduce the lowest values observed without winds (solid lines in Figs. 8 and 9), since $\alpha$ elements continue to enrich the ISM as long as the SF is not halted (a few Gyr). If there is no wind and the SF is as long as inferred form CMDs, the SF continues for a longer period and the metallicity of the ISM (and, consequently, the stellar one) continues to increase until the SF is halted, because the gas is completely consumed. As a consequence, the peak of the SMD is located at much higher $[\mathrm{Fe} / \mathrm{H}](\sim 0.0 \mathrm{dex})$ than the observed one. The SMD as a whole is shifted towards higher metallicities and entirely fails any agreement with the observed data, which strongly suggests that this scenario must be discarded (solid lines in Figs. 10 and 11). Even if the values of the main parameters of the models are changed considerably (models Leo1-nw2 and Leo2-nw2 in Table 2), the models cannot reproduce the observed data (dotted lines in Figs. 8 to 11). Only models with similar SF efficiencies as the best ones, but with very brief SF episodes ( 0.6-0.9 Gyr) can produce SMD similar to what is observed in these two galaxies. The SFH adopted in these models are however quite different from any observation: all stars form in a period shorter than 1 Gyr. The drop in the high-metallicity tails is further more instantaneous due to the sudden interruption of the SF at the epoch imposed by the burst of SF, producing a poor fit to the data. The 


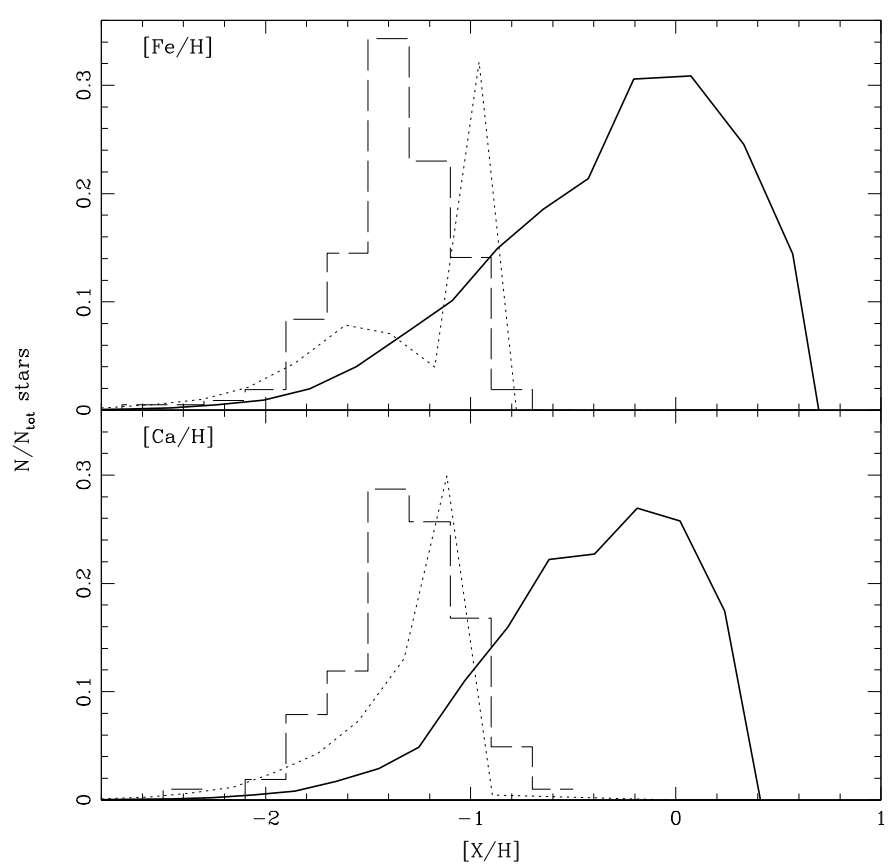

Fig. 10. The stellar metallicity distribution as a function of $[\mathrm{Fe} / \mathrm{H}]$ and $[\mathrm{Ca} / \mathrm{H}]$ observed in the dSph galaxy Leo 1 (dashed line) compared to the predictions of the chemical evolution models with no galactic winds (Leo1-nw1: solid line, Leo1-nw2: dotted line).

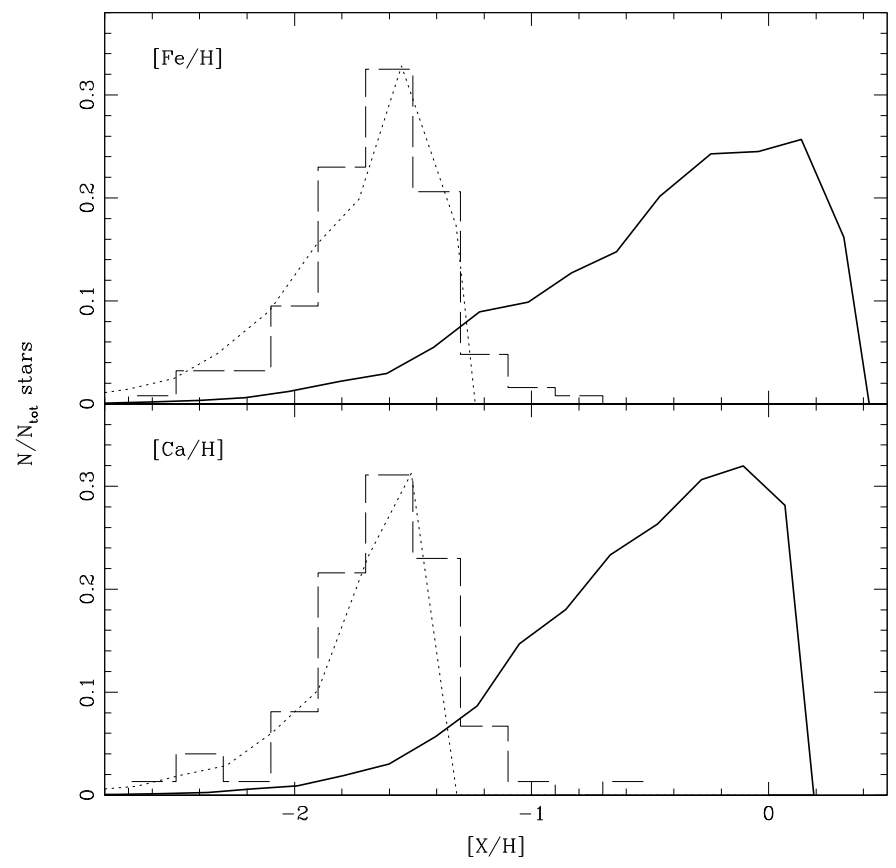

Fig. 11. The stellar metallicity distribution as a function of $[\mathrm{Fe} / \mathrm{H}]$ and $[\mathrm{Ca} / \mathrm{H}]$ observed in the dSph galaxy Leo 2 (dashed line) compared to the predictions of the chemical evolution models with no galactic winds (Leo2-nw1: solid line, Leo2-nw2: dotted line).

present day masses (gas mass and total luminous mass) also disagree with the observations: the gas mass is higher than the stellar mass (Table 2, Cols. 8 and 7 respectively) due to the low SF efficiency. It is evident then that some mechanism for gas loss must be adopted.

Therefore galactic winds as proposed in our scenario and already suggested by other authors to also explain the observed relation between $[\mathrm{O} / \mathrm{H}]$ and the mean velocity dispersions in several types of dynamically hot galaxies as well as the massmetallicity relation in dSph galaxies (Richer et al. 1998; Tamura et al. 2001), seem to be a reasonable solution.

We can summarize the main conclusions as follows:

- The main observed features of the dSph galaxy Leo 1 can be very well explained by a chemical evolution model which adopts a SF occurring in two long episodes at 14 Gyr and 9 Gyr ago, lasting 5 and 7 Gyr respectively, with a low efficiency $\left(v=\sim 0.6 \mathrm{Gyr}^{-1}\right)$ and with the occurrence of a very intense galactic wind $\left(w_{i}=9\right)$ triggered by SN explosions.

- The scenario for the evolution of Leo 2 is similar to the one of Leo 1, but with lower SF and wind efficiencies: the SF should occur in one long episode at 14 Gyr ago, lasting $7 \mathrm{Gyr}$, with a low efficiency $\left(v=\sim 0.3 \mathrm{Gyr}^{-1}\right)$, and the wind efficiency should be high $\left(w_{i}=8\right)$ to reproduce the observations.

- The predicted SMDs of both galaxies reproduce the observed ones very well. The low number of metal-poor stars is a natural consequence of the formation by slow gas infall and of the SF with low efficiency (with no need to invoke pre-enriched gas), whereas the peak at low metallicities $([\mathrm{Fe} / \mathrm{H}] \sim-1.4$ dex and -1.6 dex for Leo 1 and Leo 2, respectively) is a consequence also of the low SFR combined with the occurrence of a intense galactic wind.

- The combined effects of a low SFR and an intense galactic wind are the main responsible factors for the observed abundance ratio patterns and the features in the SMD in Leo 1 and Leo 2. The low values of $[\alpha / \mathrm{Fe}]$ and $[\mathrm{Ba}, \mathrm{Eu} / \mathrm{Fe}]$ at high metallicities, the peak at low $[\mathrm{Fe} / \mathrm{H}]$, and the sharp drop at the high-metallicity tail of the SMD are very well explained by the cessation of the SF due to the removal of gas by galactic winds.

- The comparison between the data and the predictions from a model with no galactic winds produces results at variance with observations and strongly suggests that gas loss is required to account for all observations. With no wind, the lowest values observed in the abundance ratios at high $[\mathrm{Fe} / \mathrm{H}]$ cannot be reached, and the whole SMD is shifted toward higher metallicities, at variance with the data.

Acknowledgements. We thanks Andreas Koch for providing us data of the galaxies Leo 1 and Leo 2. G.A.L. acknowledges financial support from the Brazilian agency FAPESP (proj. 06/57824-1). F.M. acknowledges finacial support from PRIN2007 MUR (Italian Ministry of University and Research) Prot.2007JJC53X-001.

\section{References}

Bellazzini, M., Ferraro, F. R., Origlia, L., et al. 2002, AJ, 124, 3222

Bellazzini, M., Gennari, N., \& Ferraro, F. R. 2005, MNRAS, 360, 185

Bonifacio, P., Sbordone, L., Marconi, G., Pasquini, L., \& Hill V. 2004, A\&A, 414,503

Bosler, T. L., Smecker-Hane, T. A., Cole, A., \& Stetson, P. B. 2004, in Origin and Evolution of the Elements, Carnegie Observatories Astrophysics Ser., 4, ed. A. McWilliam, \& M. Rauch, Pasadena

Bosler, T. L., Smecker-Hane, T. A., \& Stetson, P. B. 2007, MNRAS, 378, 318

Bradamante, F., Matteucci, F., \& D'Ercole, A. 1998, A\&A, 337, 338

Burkert, A., \& Ruiz-Lapuente, P. 1997, ApJ, 480, 297

Busso, M., Gallino, R., Lambert, D. L., Travaglio, C., \& Smith, V. V. 2001, ApJ, 557,802

Calura, F., Lanfranchi, G. A., \& Matteucci, F. 2008, A\&A, 484, 107

Carrera, R., Aparicio, A., Martnez-Delgado, D., \& Alonso-Garca, J. 2002, AJ, 123,3199

Cescutti, G., François, P., Matteucci, F., Cayrel, R., \& Spite, M. 2006, A\&A, 448,557

Cescutti, G., Matteucci, F., François, P., \& Chiappini, C. 2007, A\&A, 462, 943

Chou, M., Majewski, S. R., Cunha, K., et al. 2007, ApJ, 670, 346 
Dolphin, A. E., Weisz, D. R., Skillman, E. D., \& Holtzman, J. A. 2005, in Resolved Stellar Populations, ed. D. Valls-Gabuad, \& M. Chavez, to appear, ASP Conf. Ser., [arXiv: astro-ph/0506430]

Fenner, Y., Gibson, B. K., Gallino, R., \& Lugaro, M. 2006, ApJ, 646, 184

Ferrara, A., \& Tolstoy, E. 2000, MNRAS, 313, 291

Fragile, P. C., Murray, S. D., Anninos, P., \& Lin, D. N. C. 2003, ApJ, 590, 778

François, P., Matteucci, F., Cayrel, R., et al. 2004, A\&A, 421, 613

Freiburghaus, C., Rembges, J.-F., Rauscher, T., et al. 1999, ApJ, 516, 381

Gallart, C., Freedman, W. L., Aparicio, A., Bertelli, G., \& Chiosi, C. 1999, AJ, 118,2245

Gallino, R., Arlandini, C., Busso, M., et al. 1998, ApJ, 497, 388

Geisler, D., Smith, V. V., Wallerstein, G., Gonzalez, G., \& Charbonnel, C. 2005, AJ, 129, 1428

Greggio, L., \& Renzini, A. 1983, A\&A, 118, 217

Grevesse, N., \& Sauval, A. J. 1998, Space Sci. Rev., 85, 161

Gullieuszik, M., Held, E. V., Saviane, I., \& Rizzi, L. 2009, A\&A, 500, 735

Held, E. V., Saviane, I., Momany, Y., \& Carraro, G. 2000, ApJ, 530, L85

Held, E. V., Clementini, G., Rizzi, L., et al. 2001, ApJ, 562, L39

Helmi, A., Irwin, M. J., \& Tolstoy, E. 2006, ApJ, 651, L121

Hernandez, X., Gilmore, G., \& Valls-Gabaud, D. 2000, MNRAS, 317, 831

Koch, A., Grebel, E. K., Wyse, R. F. G., et al. 2006, AJ, 131, 895

Koch, A., Grebel, E. K., Kleyna, J. T., et al. 2007a, AJ, 133, 270

Koch, A., Wilkenson, M. I., Kleyna, J. T., et al. 2007b, ApJ, 657, 241

Lanfranchi, G., \& Matteucci, F. 2003, MNRAS, 345, 71

Lanfranchi, G., \& Matteucci, F. 2004, MNRAS, 351, 1338

Lanfranchi, G., Matteucci, F., \& Cescutti, G. 2006a, MNRAS, 365, 477

Lanfranchi, G., Matteucci, F., \& Cescutti, G. 2006b, A\&A, 453, 67

Lanfranchi, G., \& Matteucci, F. 2007, A\&A, 468, 927

Lanfranchi, G., Matteucci, F., \& Cescutti, G. 2008, A\&A, 481, 635

Mac Low, M., \& Ferrara, A. 1999, ApJ, 513, 142

Marcolini, A., D’Ercole, A., \& Brighenti, F. 2006, MNRAS, 371, 647
Matteucci, F. 1992, ApJ, 397, 32

Matteucci, F. 1996, FCPh, 17, 283

Matteucci, F., \& Greggio, L. 1986, A\&A, 154, 279

Matteucci, F., \& Tornambé, A. 1987, A\&A, 185, 51

Mighell, K. J., \& Rich, R. M. 1996, AJ, 111, 777

Monaco, L., Bellazzini, M., Bonifacio, P., et al. 2005, A\&A, 441, 141

Monaco, L., Bellazzini, M., Bonifacio, P., et al. 2007, A\&A, 464, 201

Nomoto, K., Hashimoto, M., Tsujimoto, T., et al. 1997, Nucl. Phys. A, 616, 79

Reid, M. J. 1993, ARA\&A, 31, 345

Revaz, Y., Jablonka, P., Sawala, T., et al. 2009, A\&A, 501, 189

Rizzi, L., Held, E. V., Bertelli, G., \& Saviane, I. 2003, ApJL, 589, 85

Richer, M., McCall, M. L., \& Stasińska, G. 1998, A\&A, 340, 67

Robertson, B., Bullock, J. S., Font, A. S., Johnston, K. V., \& Hernquist, L. 2005, ApJ, 632, 872

Sadakane, K., Arimoto, N., Ikuta, C., et al. 2004, PASJ, 56, 1041

Salpeter, E. E. 1955, ApJ, 121, 161

Sbordone, L., Bonifacio, P., Buonanno, R., et al. 2006, A\&A, 464, 201

Scalo, J. M. 1986, FCPh, 11, 1

Schmidt, M. 1963, ApJ, 137, 758

Shetrone, M., Venn, K. A., Tolstoy, E., \& Primas, F. 2003, AJ, 125, 684

Shetrone, M. D., Siegel, M. H., Cook, D. O., \& Bosler, T. 2009, AJ, 137, 62

Tamura, N., Hirashita, H., \& Takeuchi, T. T. 2001, ApJ, 552, L113

Tinsley, B. M. 1980, 1980, FCPh, 5, 287

Tolstoy, E., Venn, K. A., Shetrone, M., et al. 2003, AJ, 125, 707

van den Bergh, S. 1994, ApJ, 428, 617

van den Hoek, L. B., \& Groenewegen, M. A. T. 1997, A\&AS, 123, 305

Venn, K. A., Irwin, M., Shetrone, M. D., et al. 2004, AJ, 128, 1177

Wanajo, S., Tamamura, M., Itoh, N., et al. 2003, ApJ, 593, 968

Woosley, S. E., \& Weaver, T. A. 1995, ApJS, 101, 181

Woosley, S. E., Wilson, J. R., Mathews, G. J., Hoffman, R. D., \& Meyer, B. S. 1994, ApJ, 433, 229 